\title{
MAGNETIC FLUX CANCELATION AS THE BUILDUP AND TRIGGER MECHANISM FOR CME-PRODUCING ERUPTIONS IN TWO SMALL ACTIVE REGIONS
}

\section{Alphonse C. Sterling ${ }^{1}$, Ronald L. Moore ${ }^{1,2}, \&$ Navdeep K. Panesar ${ }^{1}$}

\footnotetext{
${ }^{1}$ NASA/MSFC/NSSTC, Huntsville, Alabama, USA

${ }^{2}$ Center for Space Plasma and Aeronomic Research, University of Alabama, Huntsville, Alabama, USA
} 


\section{Key Points}

- We follow two magnetically isolated small ARs, from emergenge until they produce CME eruptions.

- In both cases, the emerged polarities first spread apart, and then retract in toward each other.

- Filaments form at sites of magentic cancelation.

- Eruptions occur after further cancelation.

- These small-AR filament eruptions are similar to smaller-scale "minifilament eruptions" that produce coronal jets.

- This work, together with recent work on coronal jets, suggests that eruptions occur after $\sim 50 \%$ of a region's flux cancels. 


\section{Overview}

We follow two small, magnetically isolated CME-producing solar active regions (ARs) from the time of their emergence until several days later, when their core regions erupt to produce the CMEs. In both cases, magnetograms show: (a) following an initial period where the poles of the emerging regions separate from each other, the poles then reverse direction and start to retract inward; (b) during the retraction period, flux cancelation occurs along the main neutral line of the regions, (c) this cancelation builds the sheared core field/flux rope that eventually erupts to make the CME. In the two cases, respectively $30 \%$ and $50 \%$ of the maximum flux of the region cancels prior to the eruption. Recent studies indicate that solar coronal jets frequently result from small-scale filaments eruptions, with those "minifilament" eruptions also being built up and triggered by cancelation of magnetic flux. Together, the small-AR eruptions here and the coronal jet results suggest that isolated bipolar regions tend to erupt when some threshold fraction, perhaps in the range of 50\%, of the region's maximum flux has canceled. Our observed erupting filaments/flux ropes form at sites of flux cancelation, in agreement with previous observations. Thus, the recent finding that minifilaments that erupt to form jets also form via flux cancelation is further evidence that minifilaments are small-scale versions of the long-studied full-sized filaments.

A full report on this work appears in Sterling et al. (2018) 
Jet Model 

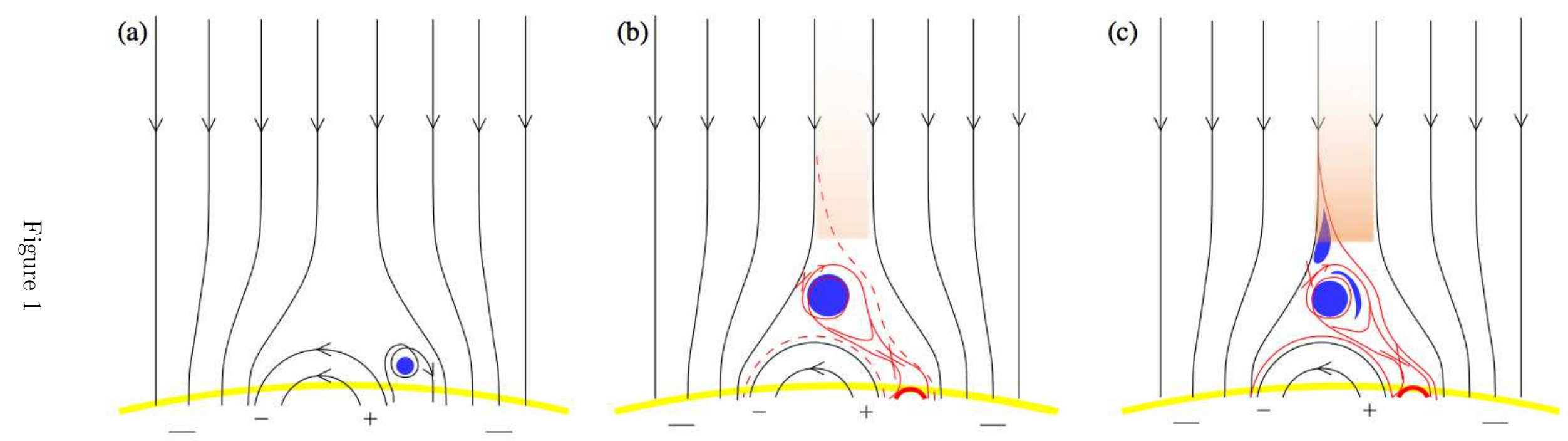

M1 M2 M3 
Figure 1. Schematic showing jet generation via a "minifilament eruption model," as proposed in Sterling et al. (2015) (with an adjustment due to Moore et al. 2018). (a) Cross-sectional view of a 3D positive-polarity anemone-type field inside of a majority negative-polarity ambient background field (which is either open or far-reaching field). One side of the anemone is highly sheared and contains a minifilament (blue circle). (b) Here the minifilament is erupting and undergoing reconnection in two locations: internal ("tether-cutting" type) reconnection (larger red $\mathrm{X}$ ), with the solid red lines showing the resulting reconnected fields; the thick red semicircle represents the "jet-base bright point" (JBP). External (a.k.a. "interchange" or "breakout" reconnection) occurs at the site of the smaller red $\mathrm{X}$, with the dashed lines indicating its two reconnection products. (c) If the external reconnection proceeds far enough, then the minifilament material can leak out onto the open/far-reaching field. Shaded areas represent heated jet material visible in X-rays and some SDO/AIA EUV channels. Labels M1, M2, and M3 point out negative, positive, and negative photospheric polarity locations, respectively. 


\section{Small-AR 1}



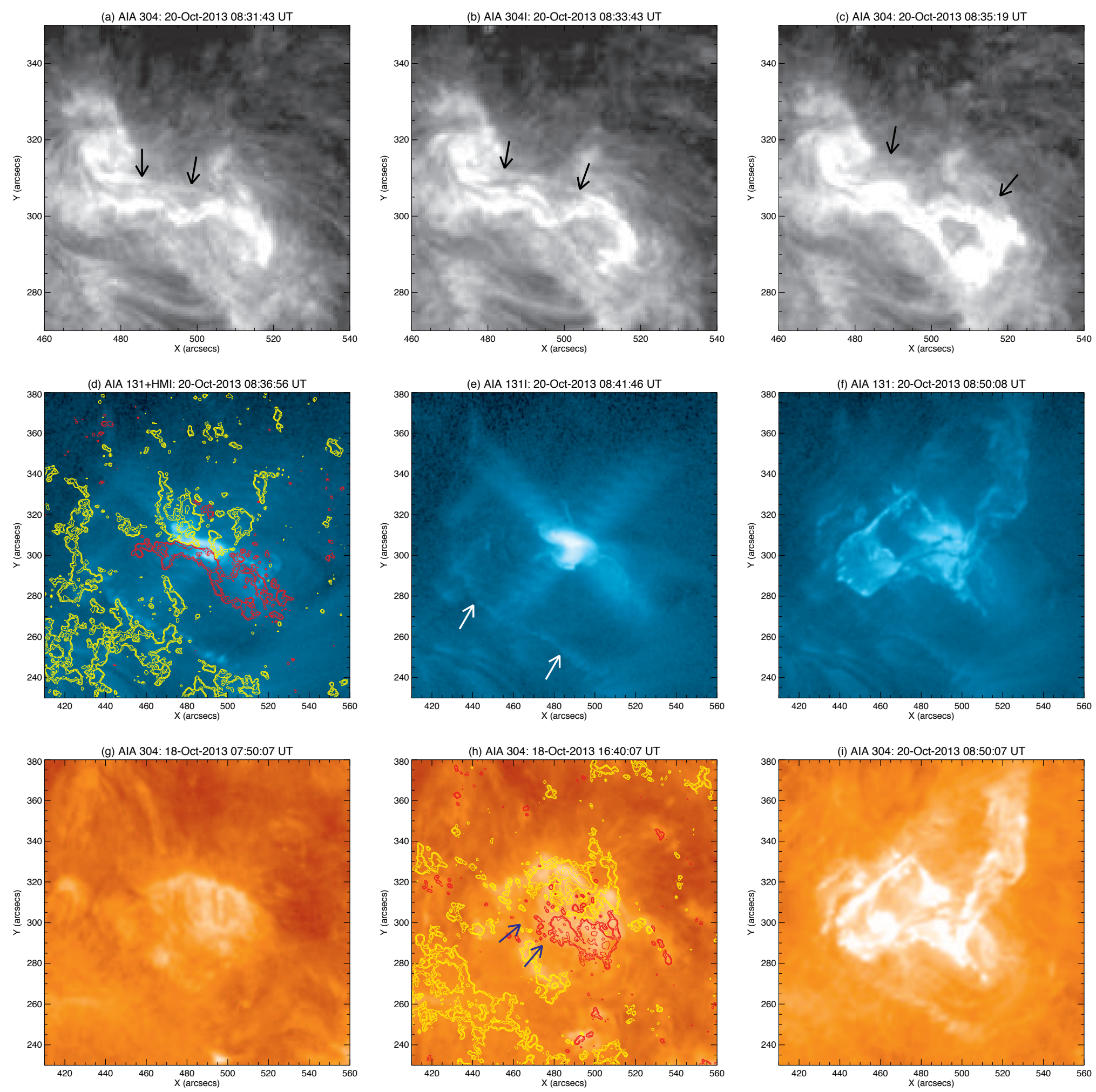

Figure 2 
Figure 2. AIA $304 \AA(\mathrm{a}-\mathrm{c} ; \mathrm{g}-\mathrm{i})$, and $131 \AA$ (d-f) images of a small- $\mathrm{AR}$ CME-producing eruption, from 2013 October. Contours in (d) are from an SDO/HMI magnetogram from 08:30 UT on October 20, and contours in (h) are from 16:45 UT on October 18, with red and yellow contours respectively representing positive and negative field. Arrows in $(\mathrm{a}-\mathrm{c})$ point out a faint flux rope erupting away from the core of the region, where the main neutral line (d) is located. Overall, the basic eruption geometry is analogous to that of the coronal jet in Fig. 1, where the flare brightening along the neutral line in (d) corresponds to the JBP in Figs. 1(b) and 1(c), and where the arrows in (e) point to "external brightenings," corresponding to the footpoints of reconnected loop fields corresponding to the location marked M1 in Fig. 1(a). Arrows in (h) point to a filament that forms along a neutral line away from the main central neutral line; this filament is erupting in (f) and (i). In these and all other solar images in this poster, north is up and west is to the right. For the event shown here, and in all other figures for this 2013 small-AR event, the images were differentially rotated to the common time of 2013 October 20, 09:00 UT. 

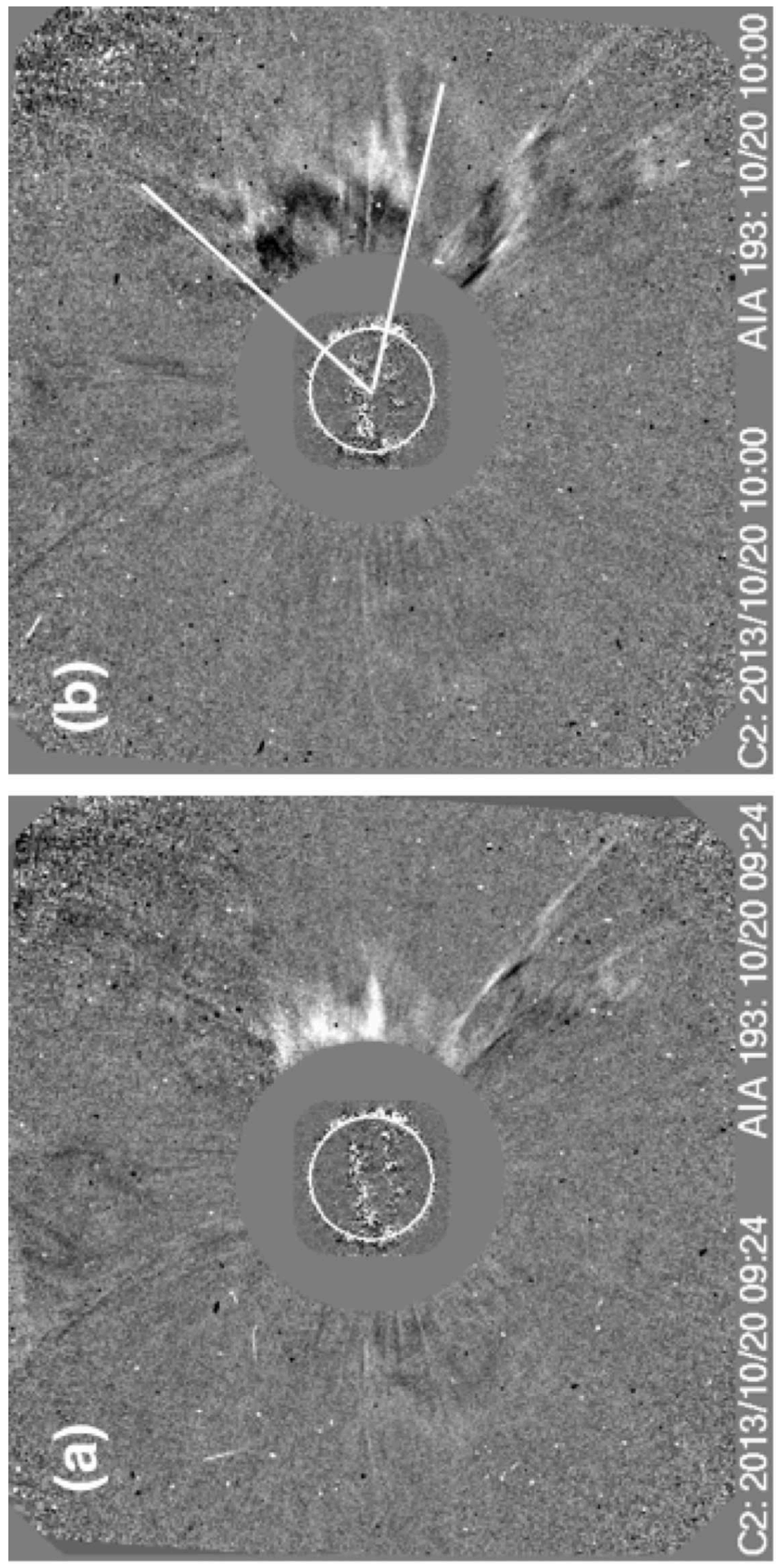

Figure 3 
Figure 3. $\mathrm{SOHO} / \mathrm{LASCO} / \mathrm{C} 2$ running difference image of the $\mathrm{CME}$ accompanying the eruption of Fig. 2. White lines in (b) subtend an angle of $\approx 60^{\circ}$. 
(a) HMI: 18-Oct-2013 02:44:45 UT

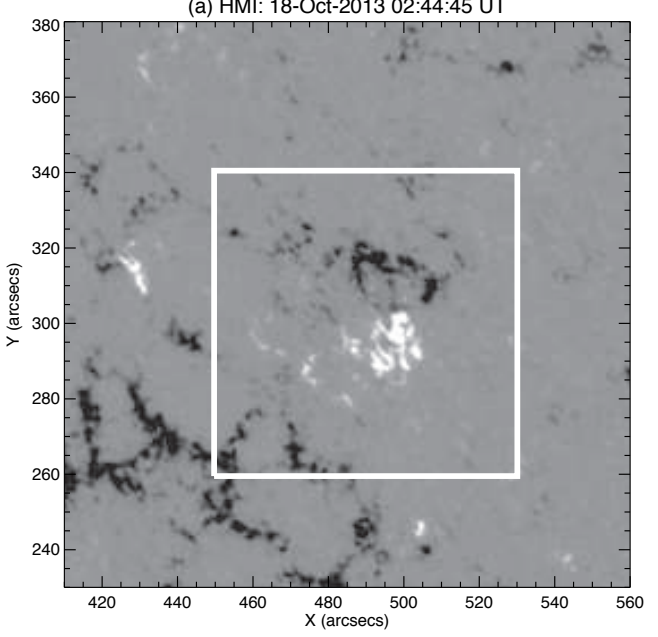

(d) HMI: 19-Oct-2013 10:29:45 UT

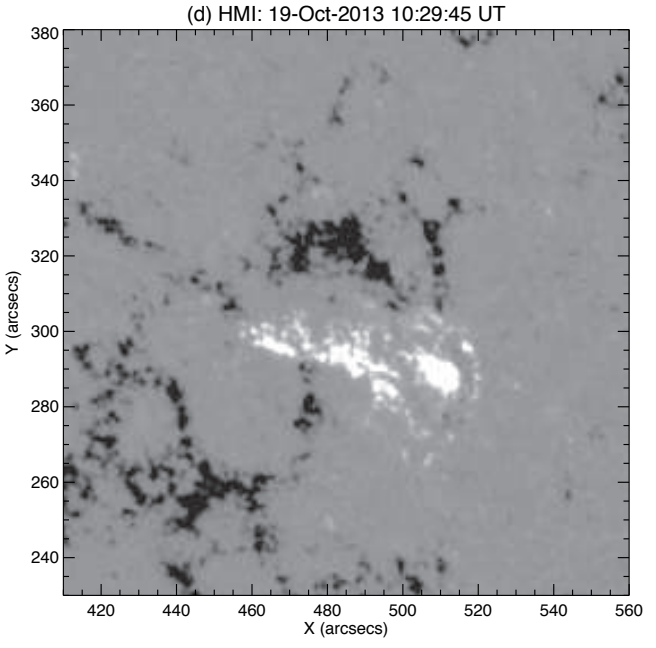

(b) HMI: 18-Oct-2013 14:44:45 UT

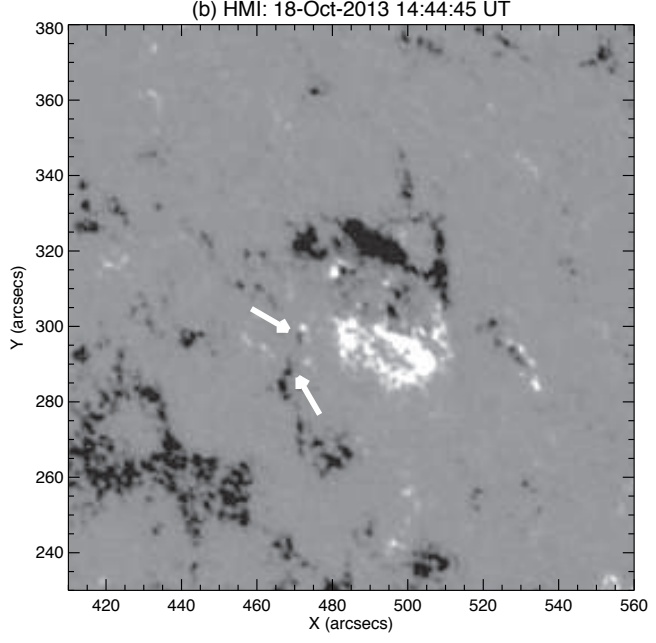

(e) HMI: 20-Oct-2013 02:29:45 UT

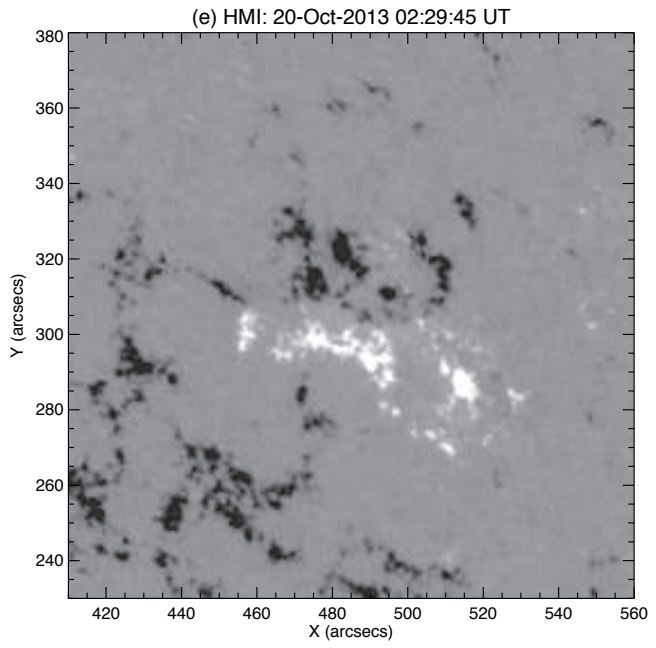

(c) HMI: 18-Oct-2013 23:59:45 UT

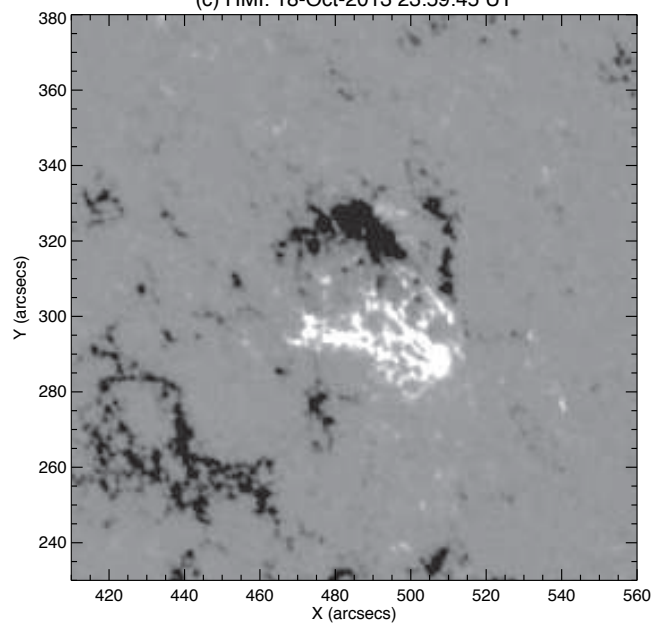

(f) HMI: 20-Oct-2013 11:44:45 UT

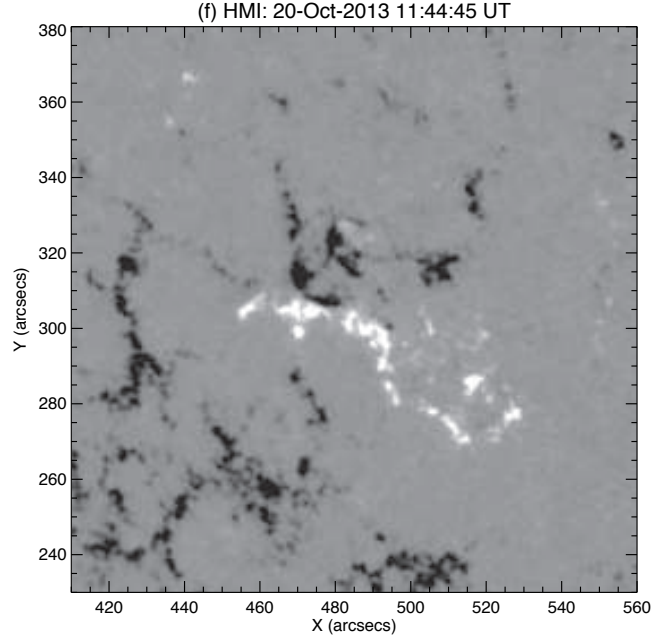

Figure 4 
Figure 4. Magnetic flux changes from HMI of the region of Fig. 2, with white/black representing positive/negative fluxes, respectively. The box in (a) shows the region used to produce the flux plot in Fig 5. Fluxes saturate at \pm 300 G. Arrows in (b) show examples of mid-strength flux elements that cancel to form the filament that erupts in Fig. 2(f). 


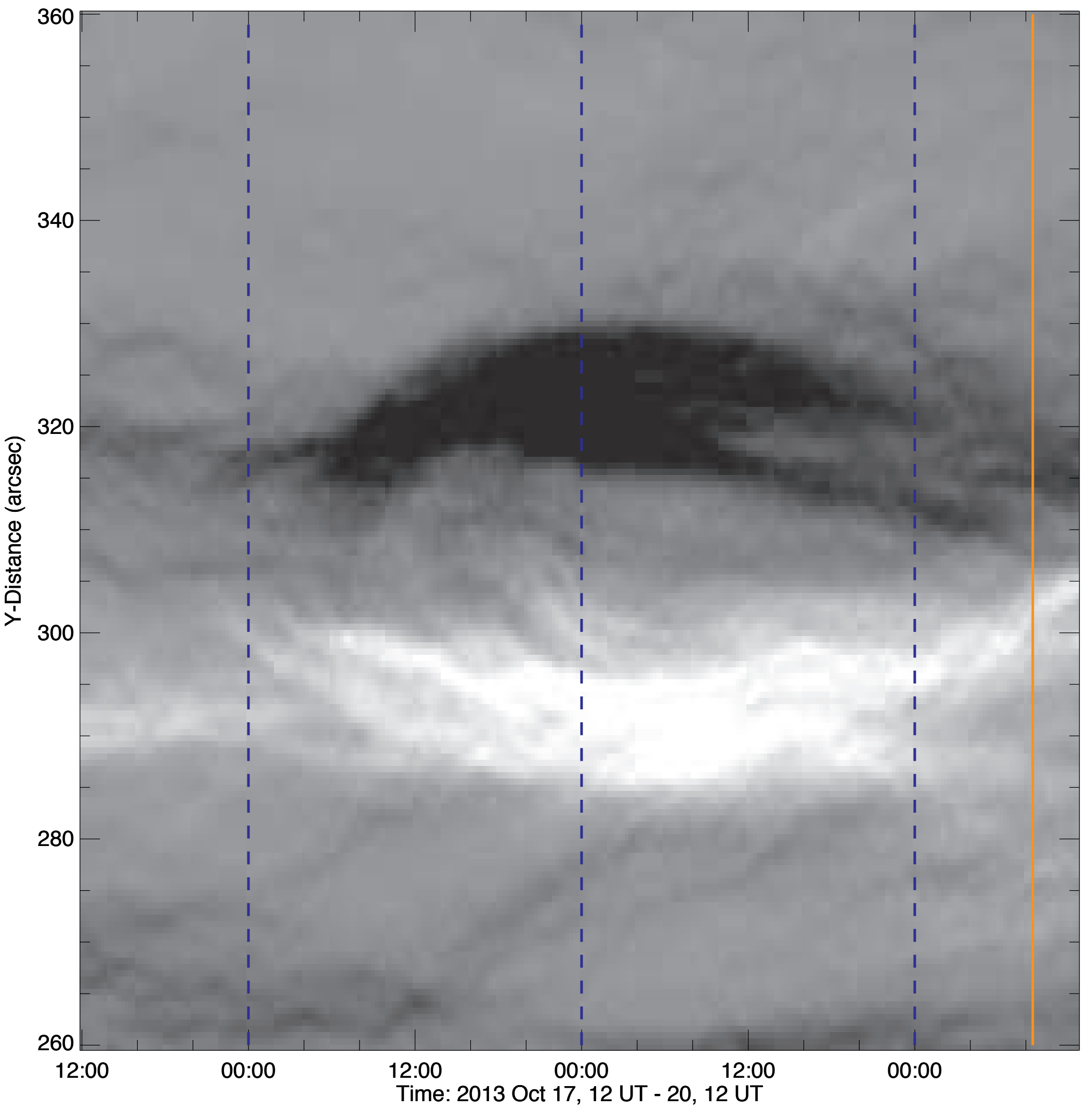

Figure 5 
Figure 5. Time-distance map for the event of Fig. 2 for the flux summed in the horizontal (E-W) direction (i.e., in pixel rows) over the box of Fig. 4(a). Flux emerges near the start; the two poles spread apart, reaching a maximum separation near 0 UT on 19 Oct; and then the two poles converge back on themselves, with cancelation occurring at the central neutral line of the bipole from approximately the start of October 20. A CME-producing eruption occurred near 08:00 UT on October 20. Blue dashed lines indicate 00:00 UT for each day, and the solid orange line shows the time of the eruption. 
Positive-Polarity Flux Change

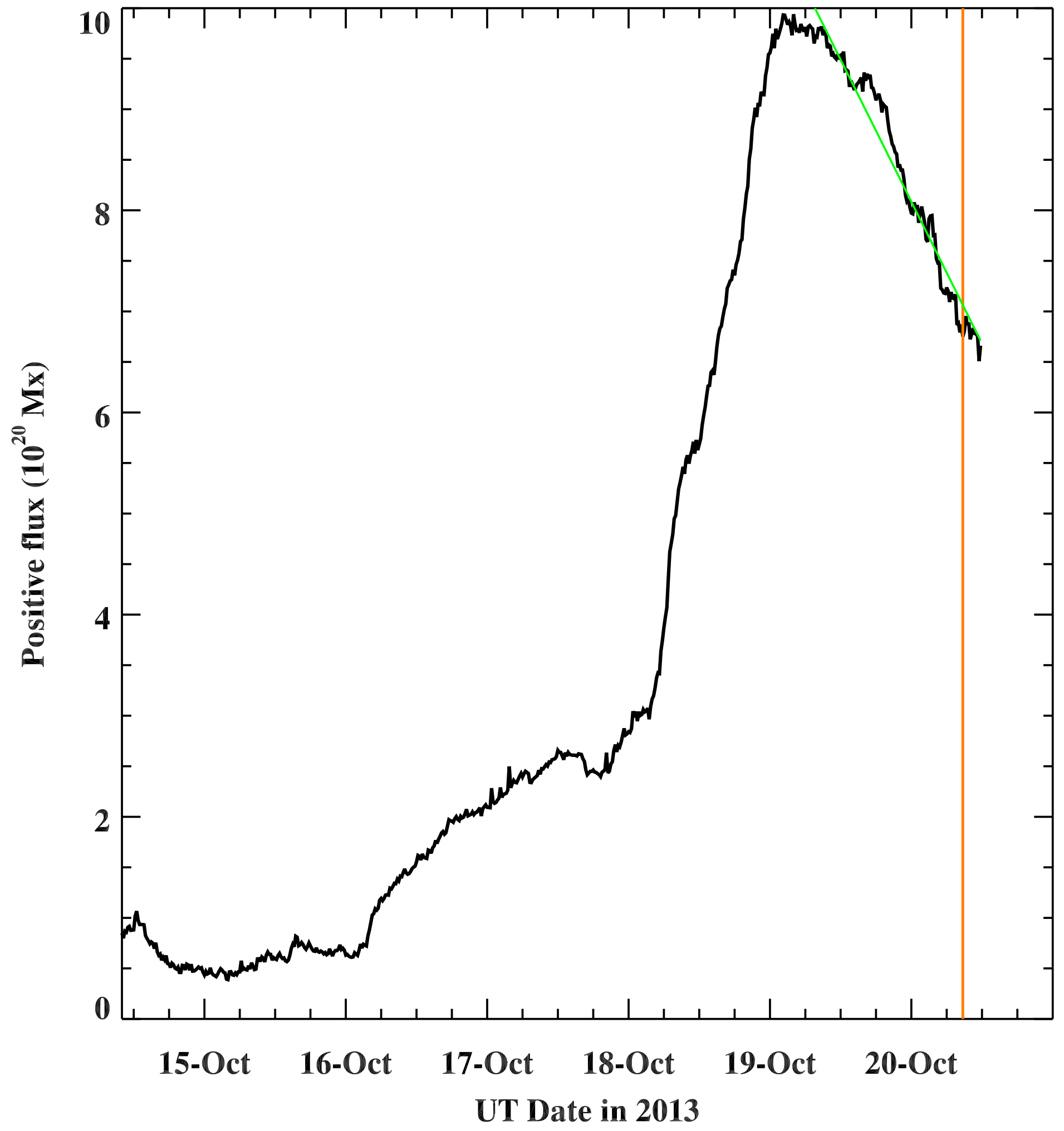

Figure 6 
Figure 6. Variation with time of positive flux for the event of Fig. 2, where the flux is the integrated positive-polarity flux over the box in Fig. 4(a). Values are calculated assuming a field component vertical to the surface, where we have approximately corrected for foreshortening by dividing by the cosine of the angle between the region and observed disk center (Sterling et al. 2018). The green line is a least-square fit over the period of the decay until the eruption (from 19 October 05:30 UT until 20 October 11:45 UT), and the orange line marks the onset time of the flare/CME-producing eruption. 
Small-AR 2 
(a) AIA 171+HMI: 16-Jul-2010 14:53:11 UT

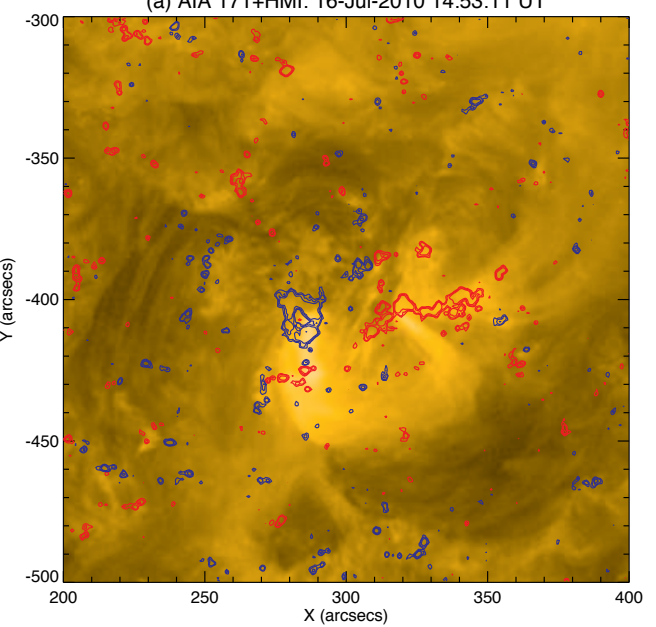

(d) AIA 304: 15-Jul-2010 12:15:02 UT

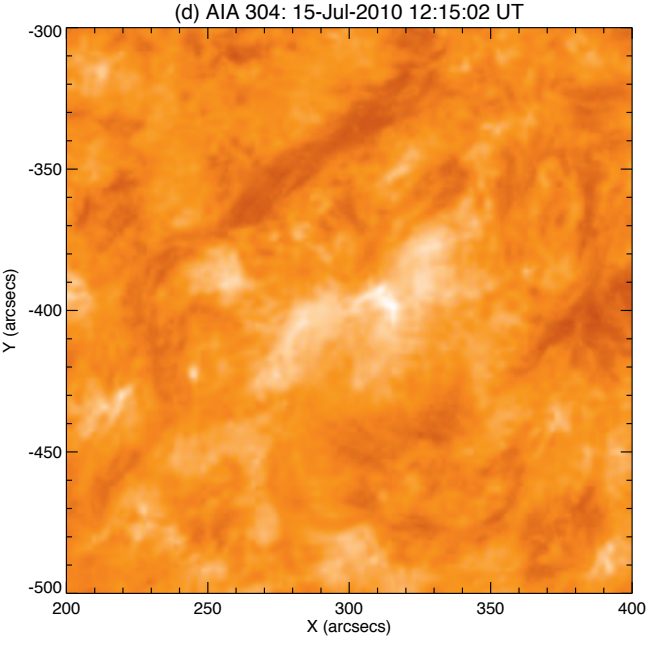

(b) AIA 171I: 16-Jul-2010 15:05:11 UT

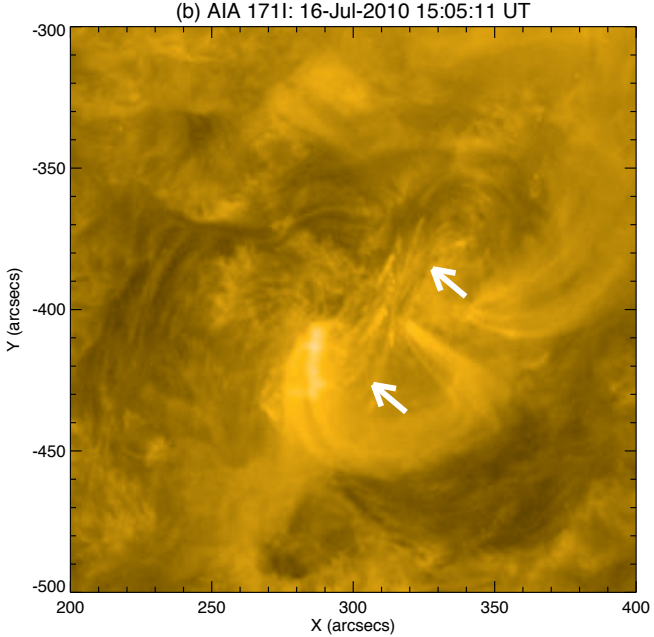

(e) AIA 304: 16-Jul-2010 09:45:02 UT

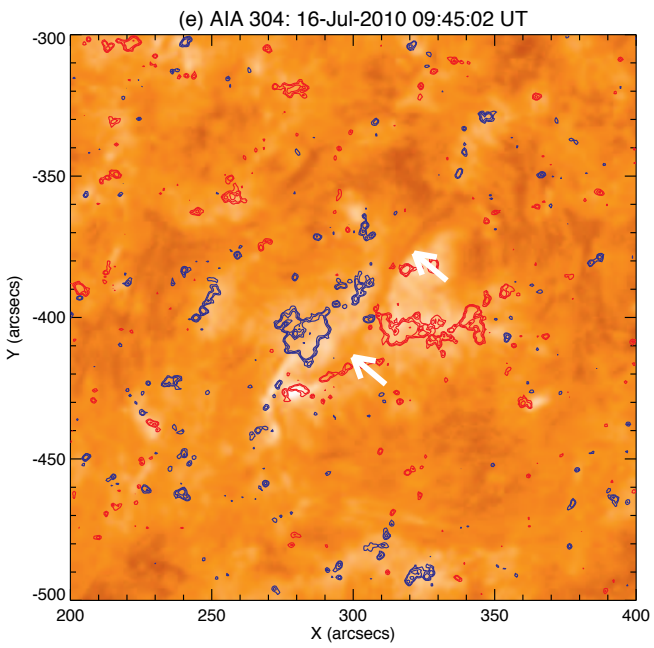

(c) AIA 171: 16-Jul-2010 15:50:11 UT

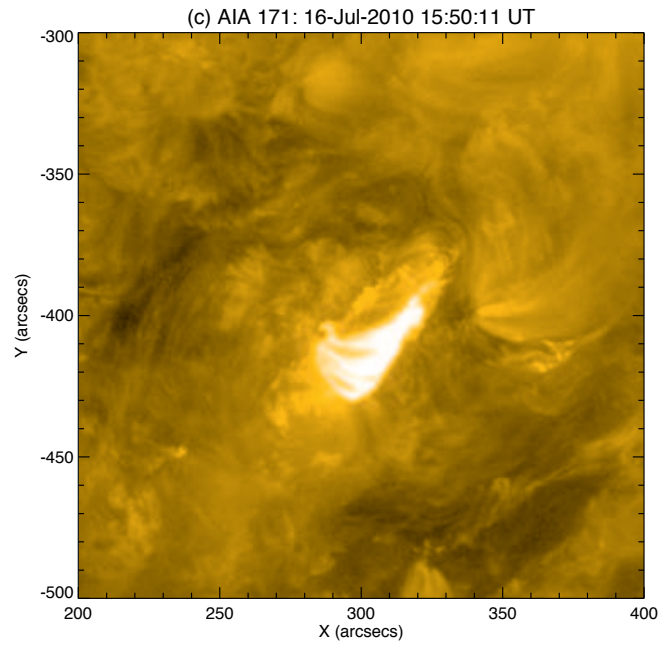

(f) AIA 304: 16-Jul-2010 16:15:02 UT

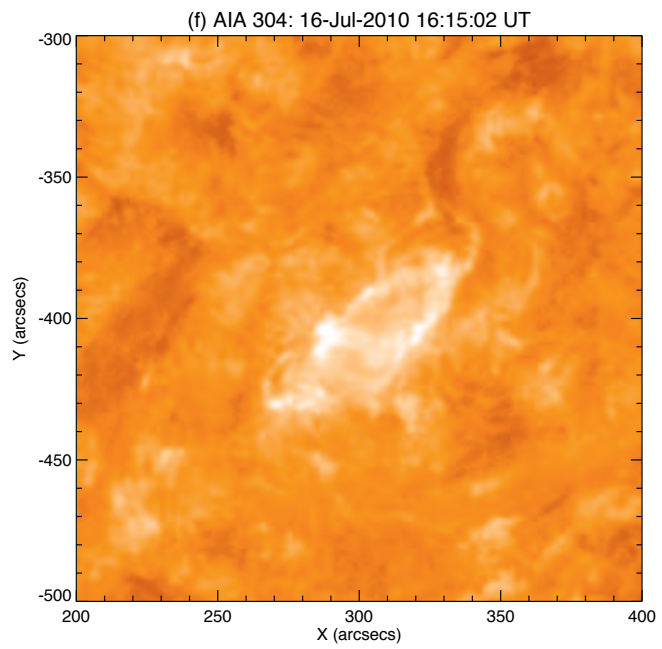

Figure 7 
Figure 7. AIA $\AA$ and $304 \AA$ images of a second small CME-producing eruption of this study, this one from July 2010. Contours in (a) are from an HMI magnetogram from 15:15 UT on July 16, and contours in (e) are from 09:45 UT on the same day, with red and blue contours respectively representing positive and negative field. In this case the filament is erupting nearly directly out of the figure; arrows in (b) and in (e) point to the filament. For the event shown here, and in all other figures for this 2010 small-AR event, the images were differentially rotated to the common time of 2010 July 16, 15:30 UT. 

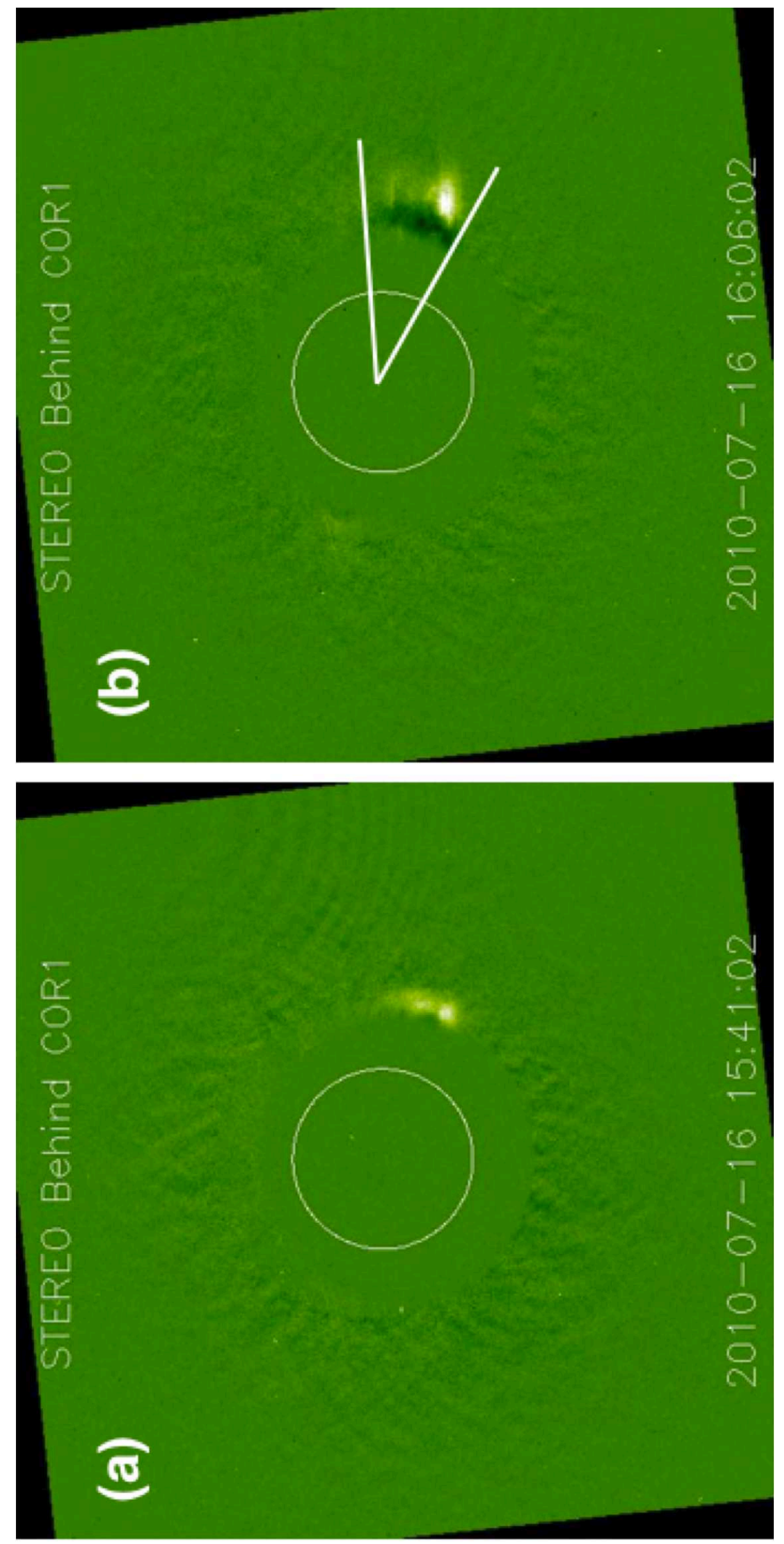

Figure 8 
Figure 8. STEREO/COR1 running difference image of the CME accompanying the eruption of Fig. 7 . White lines in (b) subtend an angle of $\approx 35^{\circ}$. 
(a) HMI: 11-Jul-2010 08:14:56 UT

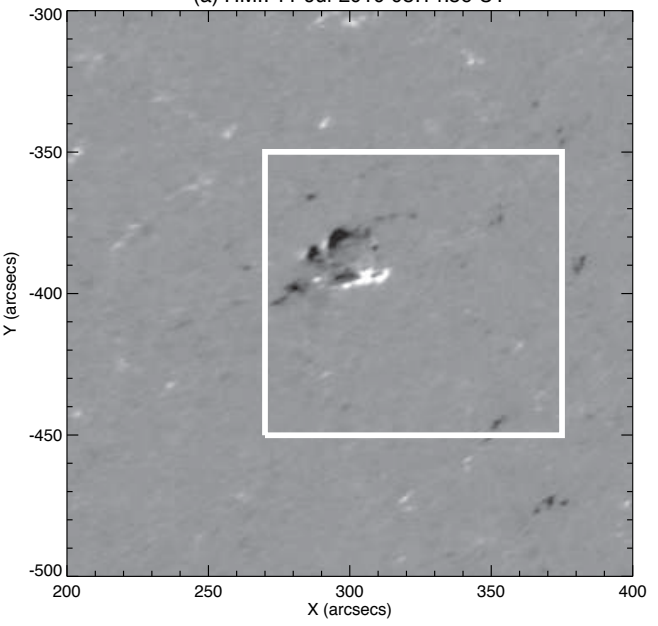

(d) HMI: 12-Jul-2010 18:59:56 UT

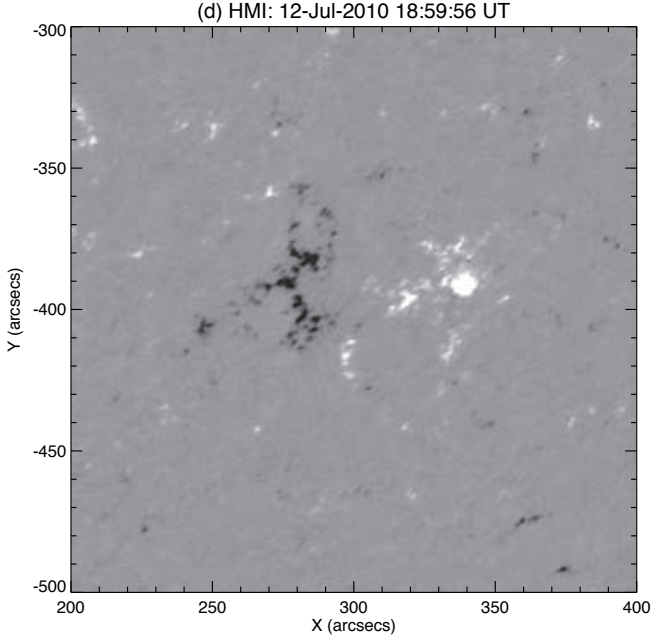

(b) HMI: 11-Jul-2010 22:14:56 UT

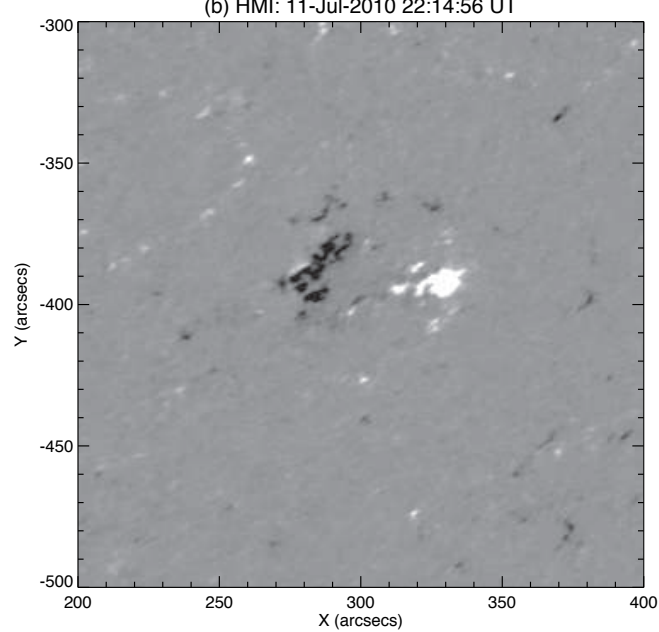

(e) HMI: 14-Jul-2010 04:14:56 UT

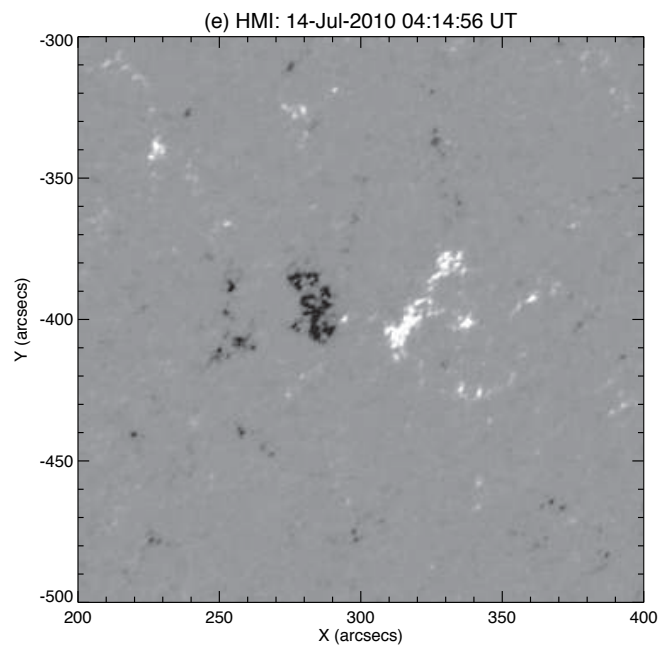

(c) HMI: 12-Jul-2010 06:29:56 UT

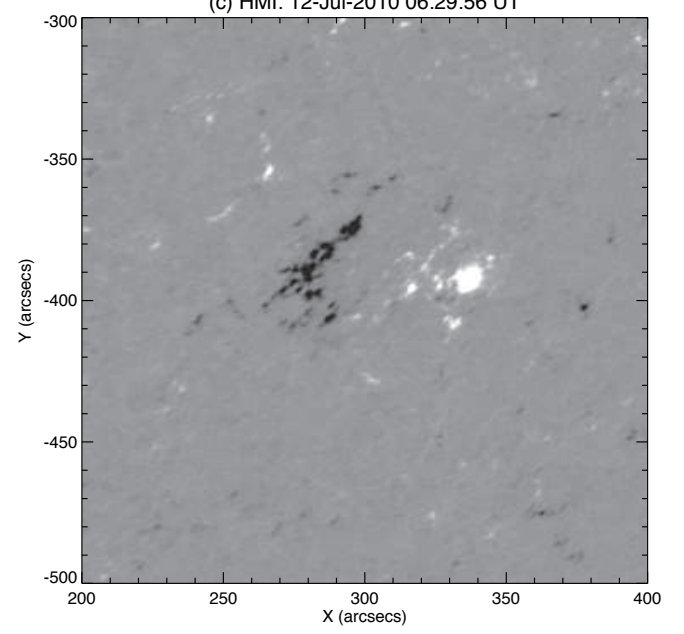

(f) HMI: 16-Jul-2010 14:59:56 UT

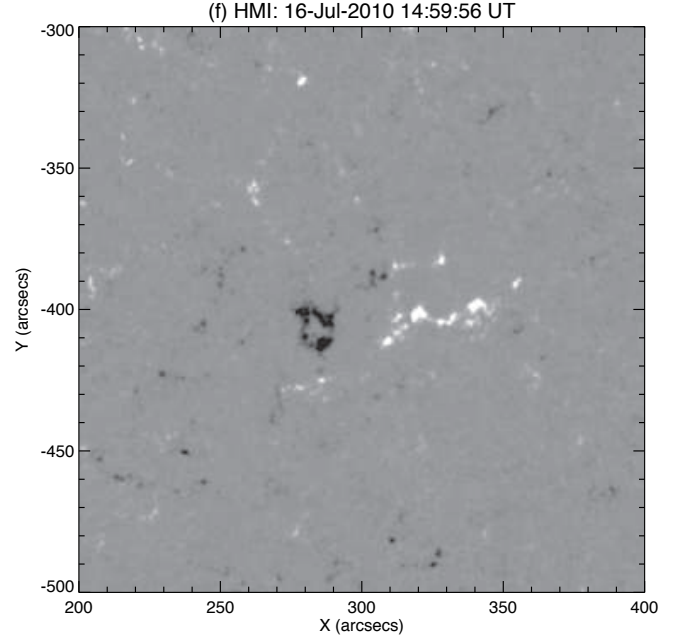

Figure 9 
Figure 9. As in Fig. 4, but for the event of Fig. 7. The box in (a) shows the region used to produce the flux plot in Fig. 9. Fluxes saturate at $\pm 300 \mathrm{G}$. 


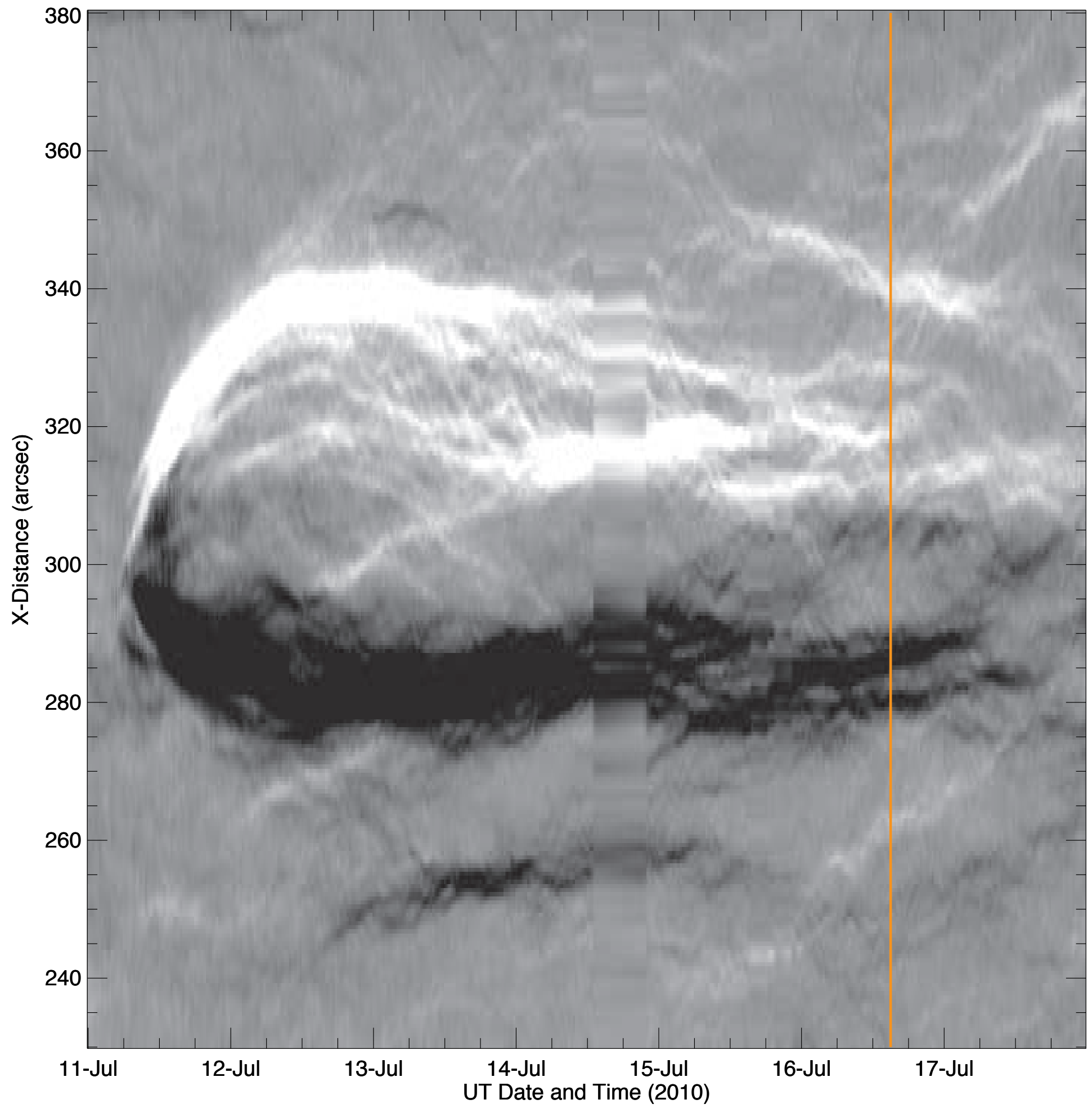

Figure 10 
Figure 10. Time-distance map, as in Fig. 5 but for the event of Fig. 7, where the flux is summed along pixel columns in the vertical (N-S) direction of the box of Fig. 7(a). The vertical strip during the last half of July 14 is an artifact resulting from $S D O$ spacecraft operations during that period, and so that portion of the map should be ignored. A CME-producing eruption occurred near 15:00 UT on July 16; the solid orange line shows the eruption time. 


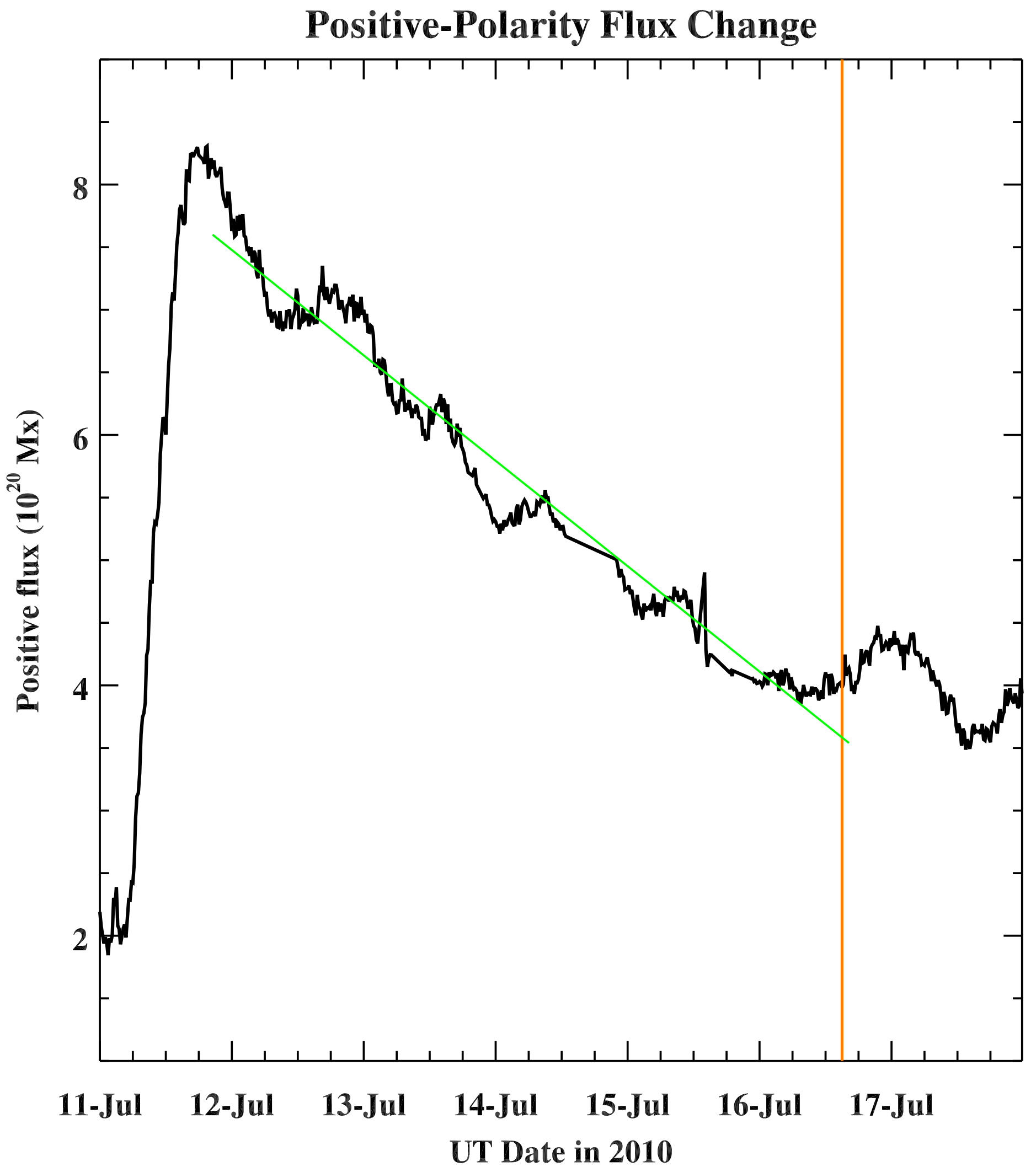

Figure 11 
Figure 11. Variation with time of positive flux for the event of Fig. 7, where the positive flux is integrated over the box in Fig. 7(a). Values are calculated assuming a field component vertical to the surface, where we have approximately corrected for foreshortening by dividing by the cosine of the angle between the region and observed disk center (Sterling et al. 2018). The green line is a least-square fit over the period of the decay until the eruption (from 19 October 05:30 UT until 20 October 11:45 UT), and the orange line marks the onset time of the flare/CME-producing eruption. 


\section{Summary and Discussion}

We have followed two different small ARs, from the time that they emerged near the solar east limb, until a time when they were the source for CME-producing eruptions. In both cases: (1) the ARs emerged, (2) their poles moved away from each other until reaching a maximum separation, (3) after that the poles started to retract in on themselves (shown well in Figs. 5 and 10), (4) after some time this retraction led to magnetic flux cancelation along the main neutral line of the emerged bipole, and (5) eventually the continued cancelation led to an eruption along the main neutral line of the retracted bipole. In the 2013 AR (first event), some of the positive flux on the outskirts of the emerging flux region canceled with nearby negative-polarity flux, and the prominent EUV filament of the region formed at that location, which was east of the main (central) neutral line of the AR. In that case, the first eruption was of a (faint in EUV) flux rope that formed along the main neutral line as a result of the cancelation of the retracting region, and that blowout core eruption apparently triggered eruption of the prominent EUV filament a few minutes later, plausibly via the Hudson effect. In the 2010 AR (second event), a filament developed along the main (central) neutral line of the emerged/retracted AR, and it was that filament that erupted to generate the CME. 
Additionally, we found evidence that filaments in both of our small ARs formed via flux cancelation. This has been found for large-scale filaments previously. More recent observations show that flux cancelation also builds minifilaments that erupt to drive jets (Panesar et al. 2017). Thus, our results here regarding filaments forming at cancelation sites also supports that there is a continuum of similarly-produced filament-like features on a variety of size scales that erupt. 
Our results suggest instead that the total percentage of flux of the entire region that cancels may be more indicative of when an eruption may occur. Although the range of values is large for the jets and the number of AR studied is small, currently the data indicate that by the time $\sim 50 \%$ of the flux of an isolated bipolar region has canceled, that region will either have already erupted or eruption is imminent. This suggests a physical explanation for the possible relationship between flux-cancelation amount and eruption onset: it could be that it is necessary for about $50 \%$ of the region's total flux to cancel for enough free energy to be built up in the flux rope (or sheared field), together with the field restraining the eruption (the non-canceled field) to be weak enough, for the eruption to take place. This suggests that, at least for regions that are sufficiently magnetically isolated from their surroundings, monitoring the change in the amount of total flux of a potentially erupting region - up to and including large CME-producing regions - could provide a prognosticator for when eruption will eventually take place. It will however be necessary to investigate additional events before drawing firm conclusions regarding these speculations. 
Table 1: Magnetic properties for coronal jets and for CME-producing small ARs:

\begin{tabular}{|c|c|c|c|c|c|}
\hline Objects & Study & Cancel Rate $\left(10^{18} \mathrm{Mx} / \mathrm{hr}\right)$ & Canceled amount ${ }^{(a)}\left(10^{18} \mathrm{Mx}\right)$ & Percentage & Energy (erg) \\
\hline CH Jets & Pucci et al. (2013) & $\underbrace{(b)}$ & $\underbrace{(b)}$ & $\underbrace{(b)}$ & $10^{26}-10^{27}$ \\
\hline CH Jets & Panesar et al. (2018) & 0.6 & $0.5-2.0$ & $45 \pm 16^{(c)}$ & $\complement^{(b)}$ \\
\hline QS Jets & Panesar et al. (2016) & $1.5^{(d)}$ & $0.9-4.0$ & $37 \pm 13^{(e)}$ & $\complement^{(b)}$ \\
\hline QS \& AR Jets $(f)$ & Shimojo \& Shibata (2000) & $\smile_{(b)}$ & $\underbrace{(b)}$ & $\underbrace{(b)}$ & $10^{27}-10^{29}$ \\
\hline AR Jets & Sterling et al. (2017) & 15 & 5 & - & $10^{28}-10^{29}$ \\
\hline 2013 Oct 20 & This poster (event 1) & 13 & 390 & $29 \pm 3^{(g)}$ & $\sim 10^{30}-10^{31}$ \\
\hline $2010 \mathrm{Jul} 16$ & This poster (event 2) & 4 & 420 & $51 \pm 3^{(g)}$ & $\sim 10^{30}-10^{31}$ \\
\hline
\end{tabular}


(a) Total average flux canceled from start of flux decline, or from time of previous events in the case of homologous jets.

(a) Total average flux canceled from start of flux decline, or from time of previous events in the case of homologous jets. (b) This quantity difficult to measure reliably, or otherwise not provided in stated study. (a) Total average flux canceled from start of flux decline, or from time of previous events in the case of homologous jets. (c) Determined using values in Table 1 of Panesar et al. (2018); see Sterling et al. (2018). (a) Total average flux canceled from start of flux decline, or from time of previous events in the case of homologous jets. (d) Data for this value are from Panesar et al. (2016), but the calculation is described in $§ 4.1$ of Panesar et al. (2018).

(a) Total average flux canceled from start of flux decline, or from time of previous events in the case of homologous jets. (e) Determined using values in Table 1 of Panesar et al. (2016); see Sterling et al. (2018).

(a) Total average flux canceled from start of flux decline, or from time of previous events in the case of homologous jets. (f) Although this study examined jets in all regions, it used Yohkoh/SXT data, which were biased toward AR and QS jets over the comparatively-softer-X-ray $\mathrm{CH}$ jets.

(g) Uncertainty determined assuming assuming flux values accurate to $2 \times 10^{19} \mathrm{Mx}$. 
A.C.S. and R.L.M. were supported by funding from the Heliophysics Division of NASA's Science Mission Directorate through the Heliophysics Guest Investigators (HGI) Program, and the Hinode project. N.K.P's research was supported by an appointment to the NASA Postdoctoral Program at NASA MSFC, administered by Universities Space Research Association under contract with NASA. 


\section{REFERENCES}

Moore, R. L., Sterling, A. C., \& Panesar, N. K., ApJ, 859, 3

Panesar, N. K., Sterling, A. C., \& Moore, R. L. 2016, ApJ, 822, 23L

Panesar, N. K., Sterling, A. C., \& Moore, \& R. L. 2017, ApJ, 844, 131

Panesar, N. K., Sterling, A. C., \& Moore, \& R. L. 2018, ApJ, 853, 189

Pucci, S., Poletto, G., Sterling, A. C., \& Romoli, M. 2013, ApJ, 776, 16

Shimojo, M., \& Shibata, K. 2000, ApJ, 542, 1100

Sterling, A. C., Moore, R. L., Falconer, D. A., \& Adams, M. 2015, Nature, 523, 437

Sterling, A. C., Moore, R. L., Falconer, D. A., Panesar, N. K., \& Martinez, F. 2017, ApJ, 844, 28

Sterling, A. C., Moore, \& Panesar, N. K. 2018, ApJ, 864, 68 